\title{
A Review of Fault Diagnosis Research on On- Load Tap-Changers
}

\author{
Liang Jin ${ }^{1, *}$, Yuqi Cai ${ }^{2}$ \\ ${ }^{1}$ College of Information Science and Engineering, Huaqiao University, 361021 Xiamen, China \\ ${ }^{2}$ College of Information Science and Engineering, Huaqiao University, 361021 Xiamen, China
}

\begin{abstract}
As the core component of transformer voltage regulation, onload tap changer plays an important role in ensuring voltage stability of power system. With the increase of times of voltage regulation, the failure rate also increases correspondingly. In view of the common faults of onload tap changer, this paper introduces several existing fault diagnosis methods and summarizes their advantages and disadvantages. Finally, the problems in fault diagnosis of on-load tap-changer and the future research focus are analysed.
\end{abstract}

\section{Introduction}

An on-load tap-changer (OLTC) is an essential part of a power transformer. It adjusts the output voltage of the transformer by changing the tap position to respond to changes in power generation and consumption. As an important voltage regulating equipment in the power system, the on-load tap-changer can automatically change the taps, adjust the transformation ratio, adjust the voltage and reduce the loss during the system operation, so it has been widely used. Through investigation and statistics of OLTC failure modes, it is found that transformer failures caused by OLTC irregular operation account for more than $27 \%$ of the total number of transformer accidents, which is the main reason for transformer failure $^{[1-3]}$. This article will summarize the research methods of OLTC fault diagnosis, and put forward the remaining problems and analysis of the links that can be broken.

\section{The extraction method of fault feature}

Since mechanical failure is the main fault of OLTC, and vibration signal contains a large amount of equipment status information, the mainstream method is based on the feature extraction of vibration signal. At present, the research focuses on vibration feature extraction and multi-fault diagnosis

\subsection{Wavelet decomposition}

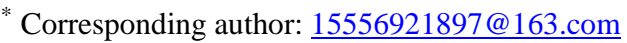


Signal denoising analysis is a pre-processing method that must be done before fault feature extraction. Here are a few simple steps ${ }^{[4]}$ :

(1) Wavelet decomposition. Calculate the high-frequency coefficients and low-frequency coefficients of each scale according to the determined optimal mother wavelet base and the optimal number of decomposition layers. The calculation formula is as follows:

$$
\begin{aligned}
& \alpha_{j, k}=<S(i), \varphi_{j, k}(\mathrm{i})> \\
& d_{j, k}=<S(i), \psi_{j, k}(\mathrm{i})>
\end{aligned}
$$

Where $j$ is the Number of layers of wavelet decomposition; $S(i)$ is the noisy partial discharge signal; $\varphi(i)$ is scale function used to calculate high frequency coefficients; $\psi(i)$ is wavelet function used to calculate low frequency coefficients.

(2) Wavelet reconstruction. Reconstructing each layer of high-frequency signals $S d j$ and the highest-scale low-frequency signals $S a j_{\max }$ using the high-frequency coefficients of each layer and the highest-scale low-frequency coefficients.

(3) Threshold denoising of signals at each scale. Threshold denoising is performed on the obtained high-frequency signals of each layer and the low-frequency signals of the highest scale, and the denoised signals are simply added to obtain the denoised waveform $S^{\prime}(i)$.

In view of the non-stationary and nonlinear characteristics of vibration signals, wavelet has its own defects in fault signal decomposition, and its decomposition effect depends on the selection of wavelet basis and decomposition scale, so it is not adaptive. In addition, there is energy leakage in wavelet decomposition. Empirical mode decomposition (EMD) has been widely used in the analysis of vibration and other non-stationary signals because of its adaptive time-frequency analysis.

\subsection{Empirical mode decomposition}

The empirical mode decomposition (EMD) method is an adaptive signal time-frequency processing method proposed by NASA Huang et al ${ }^{[5-6]}$. It has proven to be very versatile in a wide range of applications for extracting signals from data generated in noisy nonlinear and non-stationary processes. For a given signal $x(\mathrm{t})$, the EMD decomposition processes are as follows ${ }^{[7]}$ :

(1) primary selection: According to the extreme value of the signal waveform, the upper and lower envelopes are obtained by cubic spline interpolation with the maximum and minimum values, and the average $\mathrm{m}(\mathrm{t})$ of the two envelopes is obtained. And remove the average in the signal, as the initial value $h_{1}(t)$ of the intrinsic mode function (IMF).

$$
\mathrm{h}_{1}(\mathrm{t})=\mathrm{x}(\mathrm{t})-\mathrm{m}(\mathrm{t})
$$

(2) check: check if $h_{1}(\mathrm{t})$ is an IMF. If not, continue to perform the first screening process on $h_{1}(\mathrm{t})$ until the obtained component can be satisfied, then define it as IMF and record it as $F_{1}$. 
3) recycle: take as the new signal, repeat the previous two steps until all IMF components are decomposed, and define the res decomposition margin, then the original signal can be re-expressed as:

$$
x(\mathrm{t})=\sum_{i=1}^{n} F_{i}+\text { res }
$$

Although EMD has good adaptability and completeness, its modal aliasing problem is always unavoidable. Modal aliasing will not only cause distortion of the time-frequency distribution, but also make the physical meaning of the intrinsic mode function ambiguous [8-9].

\subsection{Ensemble empirical mode decomposition}

Ensemble empirical mode decomposition (EEMD) according to the statistical characteristics of Gaussian white noise, that is, a unified frequency distribution, white noise is added to the original signal, and then it is subjected to multiple EMD decompositions. The average value of the IMF component is finally used as the final result. The general steps are as follows ${ }^{[10]}$ :

(1) Add white noise to the original signal $x(\mathrm{t})$ to get a new signal $x_{j}(\mathrm{t})$, then

$$
x_{j}(\mathrm{t})=x(\mathrm{t})+\varepsilon s_{j}(\mathrm{t})
$$

Where $\mathrm{s}_{j}(\mathrm{t})$ is the $\mathrm{J}$-th addition of the white noise sequence, $\varepsilon$ is amplitude factor of the added noise;

(2) EMD $x_{j}(\mathrm{t})$ to get a set of i-layer IMF;

(3) Repeat steps 1 and 2 Ne times, and each time the white noise sequence is different;

(4) Average all IMFs by layer, that is:

$$
\overline{c_{i}}(\mathrm{t})=\frac{1}{N_{e}} \sum_{j=1}^{N_{e}} c_{i, j}(\mathrm{t})
$$

Where $c_{i, j}(\mathrm{t})$ is the $\mathrm{i}$-th layer IMF obtained at the $\mathrm{j}$-th time;

5) Finally, the decomposition result of is:

$$
x(\mathrm{t})=\sum_{i} \overline{c_{i}}(t)+\bar{r}(t)
$$

EEMD can effectively suppress mode aliasing and extract fault characteristics of vibration signals, but at the cost of computational cost. In addition, the added white noise amplitude and iteration times depend on human experience setting, and modal aliasing cannot be overcome when the value setting is not correct ${ }^{[11-12]}$. In order to find a more effective OLTC online monitoring and state assessment method that can be used in engineering practice, phase space reconstruction theory can be used to research the chaotic characteristics of vibration signals.

\subsection{Phase space reconstruction}


The theory of phase space reconstruction was first proposed by Packard [13] and Takens ${ }^{[14]}$ to study the chaotic characteristics of nonlinear signals. Through theoretical analysis and numerical calculation, it is proved that the phase space reconstruction can keep the geometric invariance of the original nonlinear dynamic system, which lays the foundation for the study of high-order nonlinear dynamic system by experimental method. As for the application of phase space reconstruction in OLTC fault diagnosis, a method based on multi-dimensional coordinate space is proposed to extract the geometric characteristics of the phase orbit diagram. By analysing the dynamic characteristics of the mechanical vibration signal of the on-load tap-changer, the specific indicators reflecting the mechanical performance are extracted. However, the phase distribution coefficient used ignores the vector characteristics of the reconstructed signal in the high-dimensional space, and can only express the relative density of the reconstructed signal., so the ability of pattern recognition for the vibration signal is limited, and there may be some unrecognized vibration signals and mis-judgments. In order to solve the above problems, some scholars use k-means clustering analysis method to identify the reconstructed vibration signal mode, and then monitor the mechanical performance of on load tap changer. The research shows that the phase trajectory obtained by the method contains abundant vibration mode information, which makes up for the difficulty of modelling the mechanical characteristics of OLTC, and provides a richer information source for the mechanical condition monitoring of OLTC. However, this method needs to establish a complete vibration fingerprint database, which can provide a basis for OLTC state evaluation and state maintenance. In addition, it is also necessary to continuously accumulate field monitoring data, analyse the changes of cluster center vector sum under the abnormal operation and typical faults of OLTC switch. Relatively speaking, the workload in the early stage will be very large. Some researchers are thinking about whether the operation state of OLTC can be judged by acoustic vibration.

\subsection{Acoustic vibration}

There are few researches on the modelling of OLTC vibration theory, feature extraction and fault diagnosis of mechanical state. Some researchers have put forward an OLTC mechanical state feature diagnosis method based on the acoustic vibration analysis technology. The vibration model of tap changer is established by the finite element analysis method. The vibration signal collected is denoised by the notch method. The vibration waveform of the switching process is intercepted by the short-time energy method. The characteristic value of the waveform is extracted by STFT based on the Blakeman window. The characteristic value data is obtained by the eigenvalue data. The reference threshold of OLTC in normal state is obtained and OLTC fault is identified. The steps of fault diagnosis based on audio are as follows ${ }^{[15]}$; first, extract useful data, normalize the amplitude of collected signal, and calculate the correlation coefficient of each tap; then resample the data, two different recording systems are used to record audio and vibration fingerprint at the same time; in addition, align the audio signal and vibration signal, and extract its envelope, that is, signal pre-processing Finally, the correlation coefficient is calculated to determine the relationship between the audio signal and the vibration signal. It should be noted that: 1 . The original signal should be pre-processed (such as signal envelope extraction); 2 . Hilbert transform acts as a low-pass filter to eliminate the fast oscillation. In addition, the Hilbert transformation can be reduced because the high frequency component in the untreated signal will cause the deviation between samples, otherwise the calculated Pearson coefficient value is much lower than the Pearson coefficient value in the signal envelope, which may lead to the false conclusion. The biggest advantage of the fault detection method based on the acoustic vibration signal is that it can monitor on-line without shutting down 
the transformer, and can carry out the fault diagnosis of the tap changer when the transformer is live. However, the fault type can't be determined, and the acoustic emission signals need to be collected continuously, and additional devices are needed, which not only increases the monitoring cost, but also takes up more space. In addition, the interference of the environment sound and the sound from the nearby machine to the audio signal is also inevitable, which needs further research on the signal acquisition device.

\section{OLTC comprehensive fault diagnosis}

The fault diagnosis expert system for OLTC mechanical properties is also applied, as shown in Figure 1. Because the diagnosis results of the on-load tap-changer fault diagnosis expert system depend on the fault symptoms, and the premise of accurately extracting the fault symptoms is that the on-line monitoring system captures the multi-channel sensor measurement signals without omission and carries out reasonable signal processing. Therefore, in addition to the artificial intelligence and calculation methods involved in the system development process, the dynamic detection closely related to the expert system. The design of data acquisition, signal analysis and other systems is also the content of fault diagnosis expert system in a broad sense.

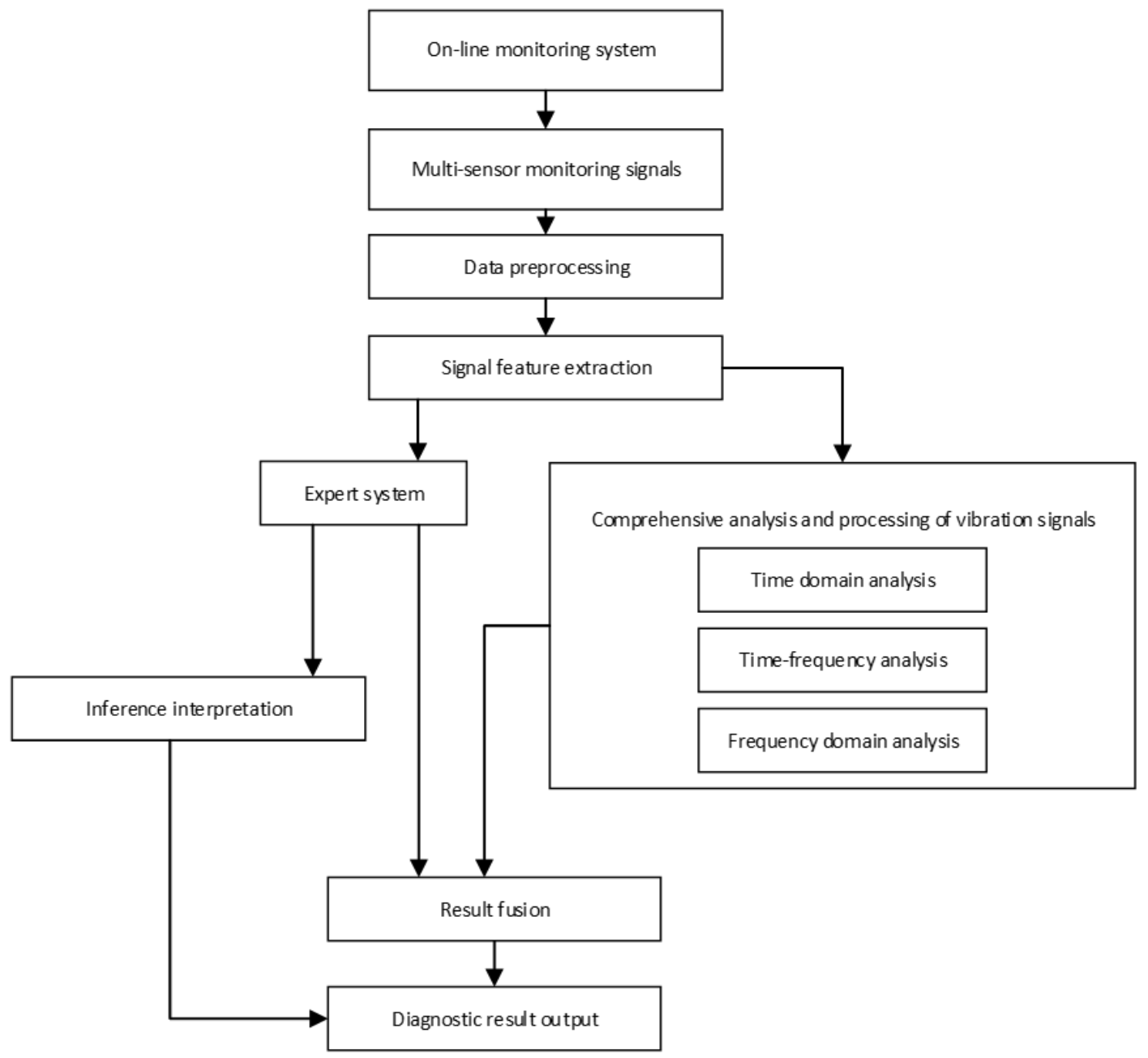

Fig. 1. Overall Structure of the Integrated Fault Diagnosis System. 
The above system can make good use of the advantages of decision tree, which is good at dealing with non-numerical data and reasoning quickly, and can also give full play to the multi angle and comprehensive analysis and diagnosis ability of vibration signal comprehensive diagnosis program. But the fault diagnosis object is only for vibration signal. However, although the fault of on load tap changer is mainly mechanical fault, there are still other faults mentioned in the first section, that is, the above system diagnosis object is not comprehensive. Therefore, in addition to the qualitative analysis of oil service test and the fault location analysis of electrical parameters, the comprehensive diagnosis of tap changer fault must also be carried out scientifically and orderly by taking various effective methods and approaches such as targeted gas chromatography analysis and electrical detection means in combination with the operation, historical data and fault characteristics of the tap changer break.

\section{Conclusion}

In this paper, several effective fault feature extraction methods are summarized, and their advantages and disadvantages are analyzed. In the mechanical fault diagnosis, the general process is shown in Figure 2:

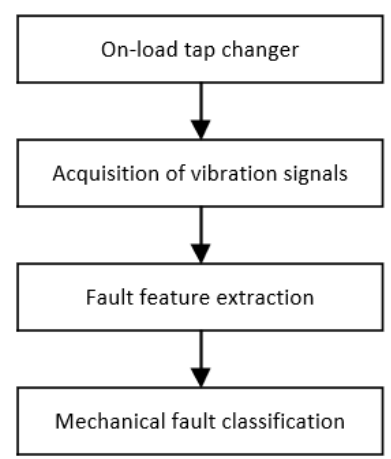

Fig. 2. Mechanical Fault Diagnosis Flow Chart with On-Load Tap-Switch.

So in the diagnosis process, we need to pay attention to the three key steps shown in Figure 2. In the process of vibration signal acquisition, attention should be paid to the position and number of sensors. If necessary, the surrounding noise should be shielded, especially based on the acoustic vibration analysis. Under the influence of the bad working environment on site, considering the antiinterference of the signal, how to design the circuit, match the amplification circuit, filter the clutter through the high-performance digital filter after the signal filtering and analog-to-digital conversion remains to be studied ${ }^{[16]}$. In the process of fault feature extraction, different methods should be selected for different signals. There are many kinds of faults in on load tap changer, and most of them are mixed faults. Different parts and different types of faults lead to different changes in the functions of the tap changer, resulting in different changes in the operation parameters of the overall and various parts of the tap changer. At present, the diagnosis method is still offline. The problems of online monitoring, the development of signal acquisition system, signal processing technology and the optimal selection of vibration measurement points need further research.

\section{ACKNOWLEDGEMENT}

This work is supported by the Subsidized Project for Postgraduates' Innovative Fund in Scientific Research of Huaqiao of University. 


\section{References}

1. J. Seo, H. Ma and T. K. Saha, IEEE Transactions on Power Delivery, vol. 32, no. 2, pp. 1031-1038, April 2017.

2. Liu JX, Wang G, Zhao T and Zhang L. ENERGIES, 2017, 10(7); $946.1-14$

3. J. Seo, H. Ma and T. K. Saha, IEEE Transactions on Power Delivery, vol. 33, no. 4, pp. 1689-1698, Aug. 2018.

4. L. Jae-Yoon, L. Dae-Jong, and J. Pyeong-Shik, Trans. Korean Inst. Elect. Eng. P, vol. 65, no. 3, pp. 188-193,2016

5. J. C. Castro, G. S. Lagos and O. A. Gonzalez, IEEE Latin America Transactions, vol. 15, no. 10, pp. 1901-1907, Oct. 2017.

6. Q. Li, T. Zhao, L. Zhang and J. Lou, IEEE Transactions on Power Delivery, vol. 27, no. 2, pp. 596-601, April 2012.

7. Coifman R R, Meyer Y, Wickerhauser V . Wavelets \& Their Applications, 1992, 9(3):765-780.

8. Huang N E. Huang, N.E. et al. Proceedings Mathematical Physical \& Engineering Sciences, 1998, 454(1971):903-995.

9. Yao J, Xiang Y, Qian S, et al. Noise source identification of diesel engine based on variational mode decomposition and robust independent component analysis[J]. Applied Acoustics, 2017, 116:184-194.

10. Wu Z, Huang N E . Advances in Adaptive Data Analysis, 2009, 01(01):1-41.

11. Packard N H, Crutchfield J P, Farmer J D, et al. Phys. Rev. Lett., 1980, 45: 712-715.

12. Takens F. Berlin: Springer, 1981.

13. Majchrzak H Cichon A and Borucki S.ARCHIVES OF ACOUSTICS, 2017, 42(1); Vol. 42, No. 1, pp. 29-35

14. A. Secic and I. Kuzle, 2018 International Symposium on Industrial Electronics (INDEL), Banja Luka, Bosnia and Herzegovina, 2018, pp. 1-6.

15. Z. Li, Q. Li, Z. Wu, J. Yu and R. Zheng, IEEE Access, vol. 6, pp. 24148-24156, 2018.

16. Roncero-Clemente C,Roanes-Lozano E.Applied Mathematics and Computation, 2017:S0096300317301285. 